\title{
Lysenin toxin insertion mechanism is Calcium-dependent
}

\author{
Ignacio L.B. Munguira ${ }^{1}$
}

U1006 INSERM, Université Aix-Marseille, Parc Scientifique et Technologique de Luminy, 163 avenue de Luminy, 13009 Marseille, France

\begin{abstract}
Pore Forming Toxins (PFTs), formed mainly by virulence factors of bacteria, belongs to Pore Forming Protein (PFP) family. Secreted as soluble monomers, they bind specific targets in membranes where their oligomerization and insertion place. Lysenin, a member of the PFTs, forms and oligomer after sphingomyelin binding, the so-called prepore, which become inserted forming a pore after a conformational change triggered by a $\mathrm{pH}$ decrease. In crowded conditions, oligomers tends to stay in prepore form because the prepore-to-pore transition is sterically blocked. In this study, we investigate the effect of calcium ions in those crowded conditions, finding that calcium act as a trigger for lysenin insertion. We localize the residues responsible for calcium sensitivity in a small $\alpha$-helix. Our results are not only one of the few complete structural descriptions of prepore-to-pore transitions but the very first that involves a calcium triggering mechanism. The presence of glutamic or aspartic acids in the insertion domains could be an indication that calcium may be a general trigger for PFTs and more generally PFP.
\end{abstract}

Keywords: pore-forming toxins; calcium; high-speed atomic force microscopy 


\section{Introduction}

Pore Forming Toxins (PFTs) ${ }^{1}$, a sub-class of Pore Forming Proteins (PFPs) family, are proteins involved in bacterial infection, among other functions. PFTs, secreted as soluble monomers in most of the cases, bind to a specific target in the membrane usually related with lipid rafts. ${ }^{1}$ After membrane binding, monomers oligomerize on the membrane, forming the so-called prepore state. The prepore-to-pore transition supposes a large conformational change and a restructuration of the membrane below ${ }^{4}$. Despite the fact that they have been studied for decades, little is known about the prepore-to-pore transition and where it takes place. To answer those two questions, it is necessary to reconstruct the life cycle of PFTs. Several studies indicate that, in PFTs from bacteria, the prepore-to-pore transition is triggered by a $\mathrm{pH}$ lower than physiological one (Bacillus anthracis, Listeria monocytogenes, Clostridium perfringens, Helicobacter pylori, Corynebacterium diphtheria and Bacillus Thuringiensis). ${ }^{5-10}$ On the other hand, calcium has also proved to regulate monomer binding activity ${ }^{11}$ and several conformational changes. ${ }^{12,13} \alpha$-Hemolysin from Staphylococcus aureus, has an aspartate acid residue which regulates the conformational change in the prepore-to-pore transition. ${ }^{14}$ In the case of lysteriolysin $\mathrm{O}$ the $\mathrm{pH}$ sensor is also formed by aspartic and glutamic acids, ${ }^{15}$ residues that are known to form part of calcium binding domains. ${ }^{16}$

Lysenin is a $33 \mathrm{kDa}$ PFT present in the coelomic fluid of Eisenia fetida. ${ }^{17}$ It belongs to the Aerolysin family which whom shares the same structure and function. ${ }^{18}$ The monomer is a soluble protein that has three domains to bind sphingomyelin-containing membranes. ${ }^{18}$ The oligomerization process, enhanced by the presence in the membrane of cholesterol, ${ }^{19}$ results in the so-called prepore state. The prepore state is a nonamer ${ }^{20}$ with a height of $90 \AA$ several Armstrongs wider than the pore, as it was shown by HSAFM. ${ }^{21-23}$ After a major conformational change that decreases the height of the oligomer $25 \AA$, the oligomer is inserted in the membrane resulting in the functional pore. ${ }^{22,24}$ The insertion is triggered by acidic conditions, ${ }^{2}$ and inhibited by crowding. ${ }^{3}$

\section{Results}

In this study we use HS-AFM to study the conformational change of prepore-to-pore lysenin transition on supported lipid bilayers (SLB) composed of SM/Chol 1:1. Incubation at high density of lysenin monomers gives as a result an hexagonal close packed (hcp) assembly (2D crystal) with $12.8 \pm 3.9 \%$ of oligomers in pore state (Figure 1a). When incubating at high monomer density in a buffer with $5 \mathrm{mM} \mathrm{CaCl}_{2}$ we observe an increase in the percentage of pore state oligomers (Figure $1 \mathbf{b}$ ), being the pores the $20.8 \pm 3.5 \%$ of the total number of oligomers. Incubation at low density of lysenin results in an amorphous assembly with $77.6 \pm 2.3 \%$ of oligomers in pore state surrounded by membrane free of oligomers (Figure 1c). We observe that monomer density and the presence of calcium during the incubation drive the pores/prepores ratio in the oligomer population (Figure 1d). This could indicate that the prepore-to-pore transition, blocked 
by steric interactions in the hcp structure, is partially overcome by physiological concentration of calcium. ${ }^{27}$
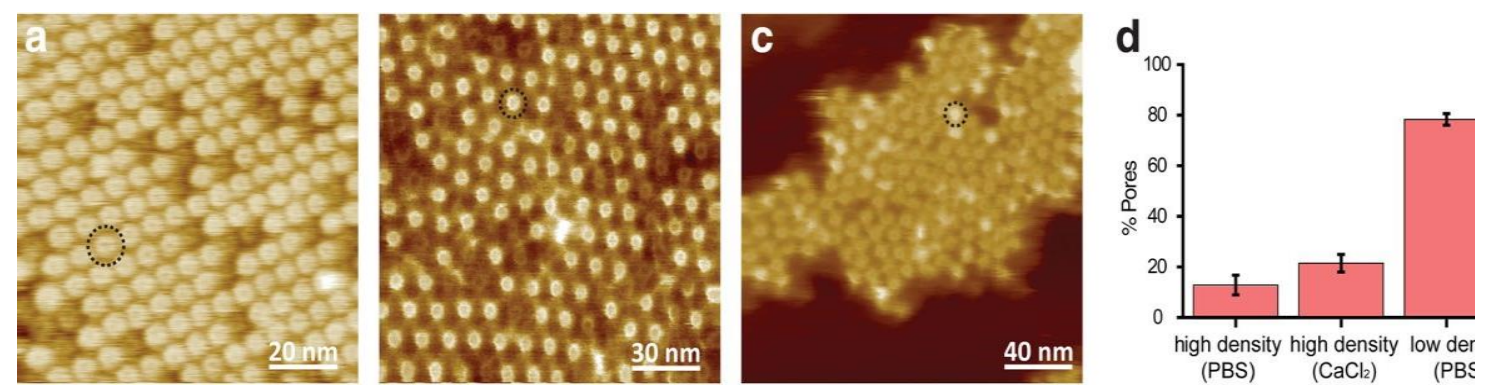

Figure 1 | Pores proportion as a function of protein incubation conditions. a) Frame of a HS-AFM of Lysenin in SM/Chol 1:1 membrane incubated at high density of Lysenin in PBS. Z scale $=0-7.9 \mathrm{~nm}$ b) Frame of a HS-AFM of Lysenin in SM/Chol 1:1 membrane incubated at high density of Lysenin for one hour in physiological buffer with calcium ions. Z scale $=0-6.17 \mathrm{nmc}$ ) Frame of a HS-AFM of Lysenin in SM/Chol 1:1 membrane incubated at low density of Lysenin in PBS. Z scale=0-16.2nm d) Pore percentage as a function of the incubation conditions. Three Lysenin prepores are highlight with a dash circle for clarity.

Taking advantage of the steric block found in lysenin at high monomer incubation to elucidate the effect of calcium cations on the prepore-to-pore transition, we prepare a Sphingomyelin (SM)/Cholesterol (Chol) 1:1 membrane in which we incubated lysenin at high monomer concentrations in order to recreate a situation with a majority of lysenin oligomers in the prepore state, highlight by an Standard Deviation map (SD-map) of every frame from the video (Figure 2a). Under those conditions with a steric block of the prepore-to-pore transition, we test the effect of calcium cations as trigger of the insertion conformational change. While recording an HS-AFM movie (Supplementary video 1), we increase the calcium concentration from 0 to $65 \mathrm{mM}$ observing a massive insertion of prepores (Figure $\mathbf{2 b}$ ).

After observing the effect of calcium in the prepore-to-pore conformational change we decided to study if the role of calcium is exclusively related with its charge, using magnesium to test it. In order to test the effect of magnesium in a SM/Chol 1:1 membrane with a majority of prepores (Figure 3a), we increase step-by-step magnesium concentration, while HS-AFM image acquisition, up to $150 \mathrm{mM}$ (Figure 3b). After reaching $150 \mathrm{mM}$ of magnesium concentration we did not observe any substantial effect on the percentage of pores, as is clearly shown by the z SD-map (Figure 3c). 

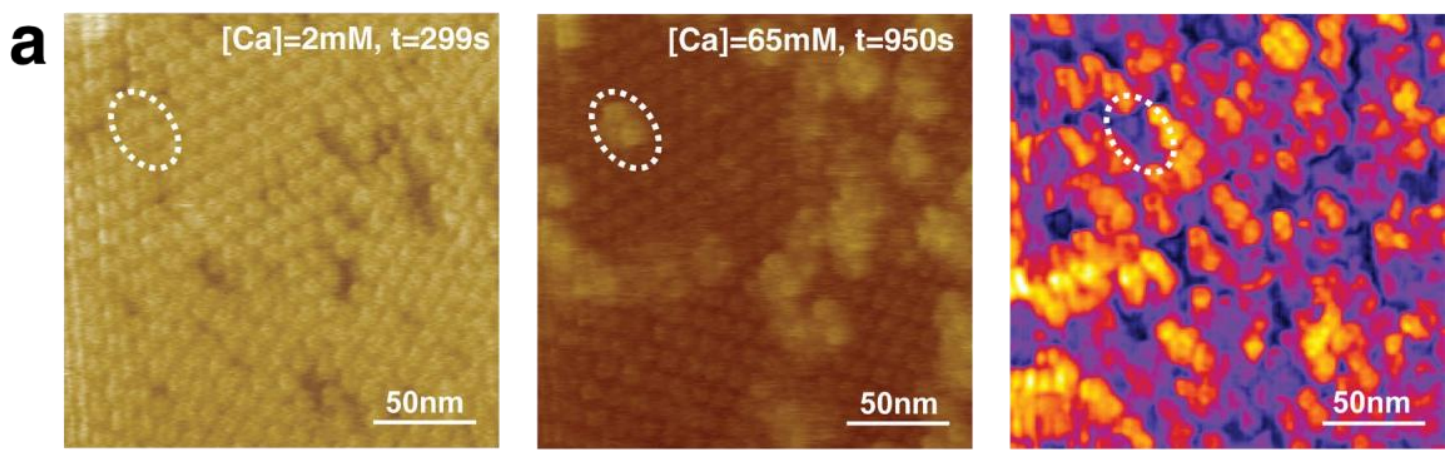

b

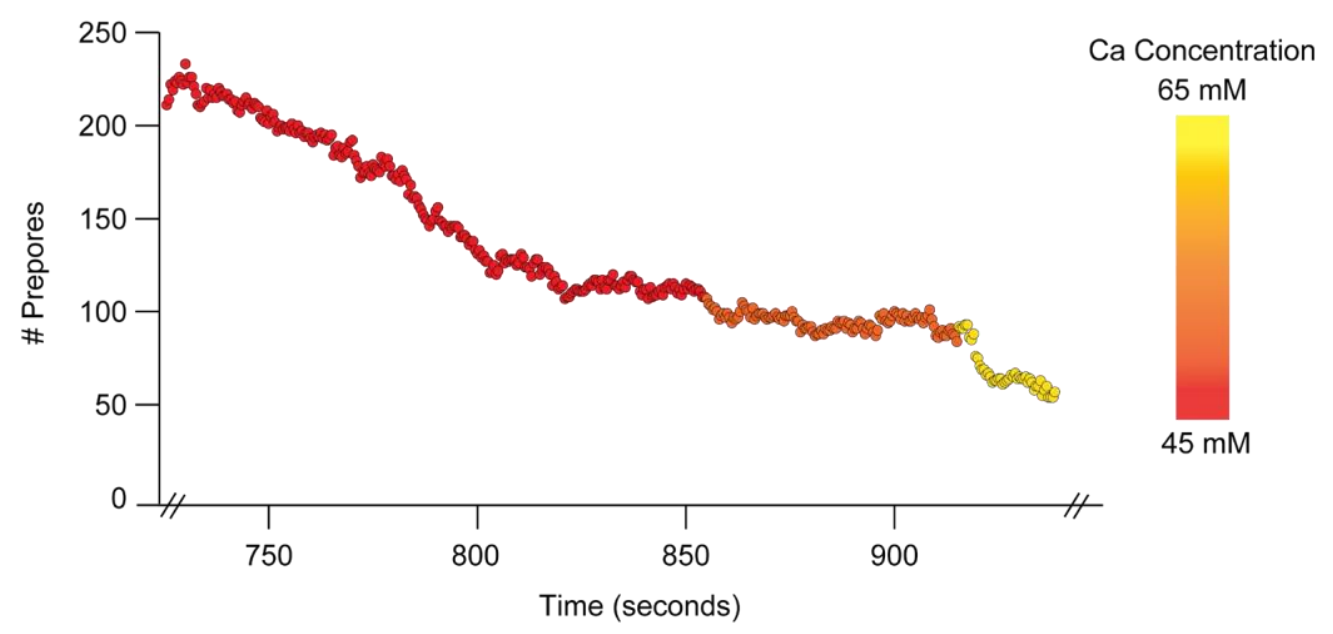

Figure 2 | Lysenin toxin membrane insertion is Calcium dependent. a) HS-AFM movie frames of lysenin ( $t=299 \mathrm{~s}, \mathrm{t}=950 \mathrm{~s}$ ) on a sphingomyelin/cholesterol (1:1) bilayer (Supplementary movie 1), and Standard Deviation map (SD-map) of the entire movie. During HS-AFM movie acquisition, the calcium concentration was increased from 0 to $65 \mathrm{mM}$ leading to an increased number of prepore lysenins (bright dots, protruding $\sim 2.9 \mathrm{~nm}$ ) inserting into the membrane to become pore lysenins (dark dots, protruding $\sim 1.3 \mathrm{~nm}$ ). b) Graph of the number of prepores (i.e. image area occupied by pixel $>113 \mathrm{~nm}$ ) as a function of movie acquisition time. The precise number of prepores and pores has been analyzed by cross-correlation searches at six specific time points (dashed lines) corresponding to the frames shown in a) $z$ range $=0-5.2 \mathrm{~nm} . \mathrm{SD}=0.2-1.3 \mathrm{~nm}$

We structurally study the origin of the calcium effect on lysenin insertion analyzing the sequence and structure of lysenin looking to a region liable to be a calcium sensor domain. We identify a small $\alpha$-helix in the Pore Forming Module (PFM) ${ }^{4}$ with a glutamic acid cluster that could be responsible for the calcium sensitivity owing its negative character and the need of this $\alpha$-helix to be detached from the rest of the structure to form the $\beta$-barrel. To proof if our speculation is valid we mutate the glutamic acids of this region (Glu 92, 94, 97) into glutamines neutralizing, therefore, the negative charge of the region. This mutant will be refer as lyseninQ for now on. We image an hcp assembly of lyseninQ with a majority of prepores, as we did with wild-tipe lysenin, increasing the calcium concentration from zero to $200 \mathrm{mM}$, threefolds the maximum calcium concentration used for the wild-type lysenin (Figure 4a). LyseninQ shows a discrete response to an increase in calcium (Figure 4b). Therefore, lyseninQ keeps the 


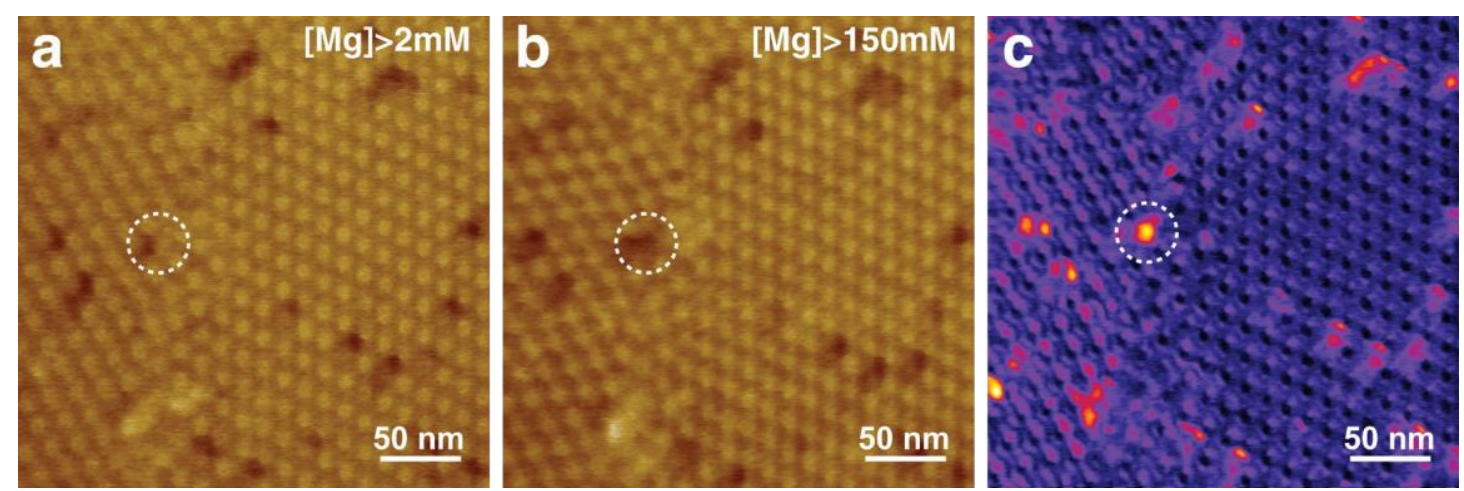

Figure 3 | Lysenin toxin membrane insertion is not Magnesium dependent. HS-AFM movie frames of lysenin on a sphingomyelin/cholesterol (1:1) bilayer (Supplementary movie 2). During HS-AFM movie acquisition, the magnesium concentration was increased from 0 to $167 \mathrm{mM}$ adding $\mu$ l of a solution with $\mathrm{MgCl}_{2}$. a) HS-AFM frame at $\mathrm{t}=103 \mathrm{~s}$. The magnesium concentration is $4 \mathrm{mM}$.b) HS-AFM frame at $\mathrm{t}=383 \mathrm{~s}$. The magnesium concentration is $167 \mathrm{mM}$. The dash circle indicate the only prepore that gets inserted along the experiment, the prepore is mark in a) also with a dash circle. c) Standard deviation map $z$ range $=0-5.7 \mathrm{~nm}$ SD range $=0.3-1.2 \mathrm{~nm}$

Insertion ability; however, it loses its calcium sensitivity as clearly shown by the $z$ standard deviation map (Figure 4c). The two observed insertions could be triggered by the high calcium concentration, approximately three-folds the concentration needed for the wild-type lysenin, but we could not discard a triggering of a different nature owing to the extremely high calcium concentration.
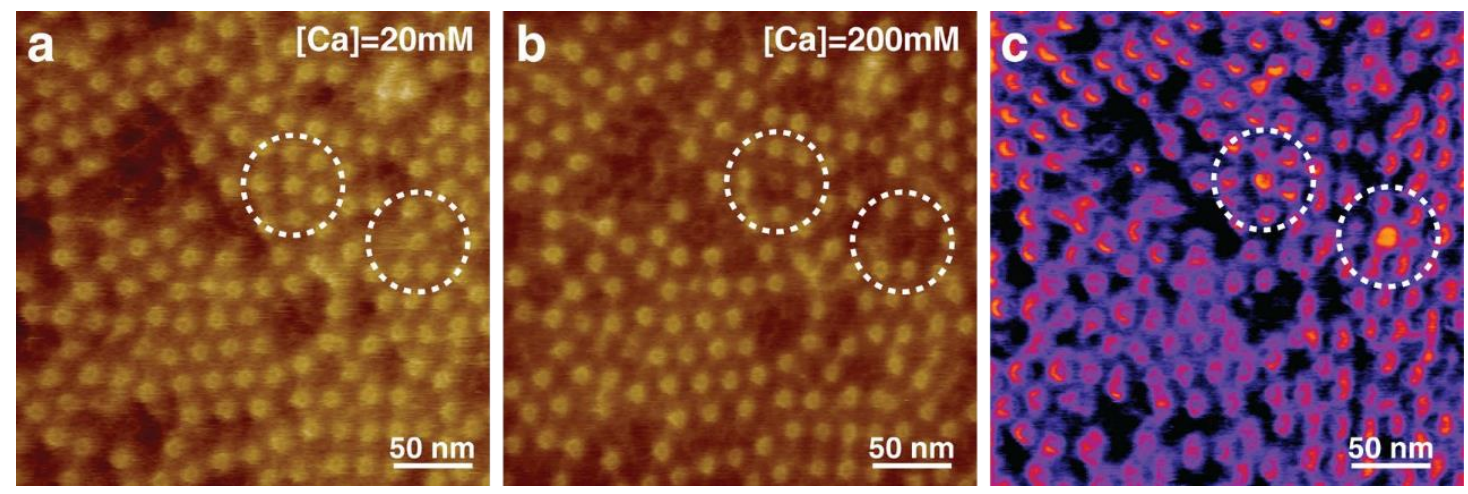

Figure 4 | The lysenin toxin pH-sensor mutant is insensitive to calcium. HS-AFM movie frames of lyseninQ mutant on a sphingomyelin/cholesterol (1:1) bilayer (Supplementary movie 3). During HSAFM movie acquisition, the calcium concentration was increased from 0 to $200 \mathrm{mM}$ a) HS-AFM frame at $t=63 \mathrm{~s}$. The calcium concentration is $20 \mathrm{mM}$. b) HS-AFM frame at $t=568 \mathrm{~s}$. The calcium concentration is $200 \mathrm{mM}$. The dash circles indicate the two prepores that get inserted along the experiment. c) Standard deviation map. The two prepores that undergo a conformational change to the pore state are in the center of the hexagon surrounded by a dash circle. The SD-map indicate that the change in the calcium concentration did not affect the lyseninQ insertions significantly. $z$ range $=0-6.67 \mathrm{~nm}$ SD range $=0.2$ $0.8 \mathrm{~nm}$ 


\section{Discussion}

In this work we have presented the effect of calcium cations as trigger of the activation mechanism of lysenin prepore in the conformational change that ends with an oligomer in the pore state. To study the prepore-to-pore transition, it has been a successful strategy to block the prepore state mutating certain regions..$^{25,26}$ On the other hand, recent findings on the prepore-to-pore transition blocking by crowding allow us to study the role of different cation on the prepore-to-pore transition. We have first observed an increase in the number of pores at a physiological calcium concentration. An increase calcium concentration 0-65 mM, while we record a HS-AFM movie, observing a sudden and massive number of insertions. In view of those results, we speculate that divalent cations trigger lysenin insertion; and we have performed an equivalent experiment with magnesium. However, from our experiment we can conclude that magnesium does not have a significant effect as a trigger of lysenin insertion. This difference between magnesium and calcium should be a manifestation of the high coordination flexibility and polarizability of calcium that allows calcium to interact effectively with complex molecules of irregular geometry as proteins. ${ }^{27}$ We have analized the structure of lysenin monomer in search of a negative region involved in the conformational change that results in the $\beta$-barrel. We identify an $\alpha$-helix that contains a glutamic acid cluster as good candidate to be a sensor for two reasons; first, the region belongs to the PFM and should be unfolded prior to the formation of the $\beta$-barrel; second, the high concentration of negative residues ensures a rich electrostatic response. Therefore, we have mutated three glutamic acids in that region (Glu92, Glu94 and Glu97) into glutamine, annulling any electrostatic reactivity. As a result of this mutation lysenin loose calcium sensitivity without losing its insertion ability. We hypothesize that calcium with its high polarizability and coordination flexibility may participate in the interaction via bound $\mathrm{Na}^{+}$of Glu92 and Glu71 creating a repulsion that unfolds the $\alpha$-helix like the leaf of a hinge (Figure 5). We observed that a similar chaotropic mechanism could also operate for the rest of the aerolysin family involving Glu252-Asp193 for aerolysin, Glu225-Asp305 for Hemolytic lectin, Glu183-Asp126 for Parasporin-2 and Glu137Asp250 for $\varepsilon$-toxin (Figure 5). A calcium activation could be behind the activation mechanism of lysteriolysin O mediated by Glu247, Asp208 and Asp320 also coordinated by $\mathrm{Na}^{+}$; Anthrax Asp425 can also be affected by calcium cations.

Gathering all these facts, we propose a cycle of lysenin pore formation mechanism, in which lysenin would follow two different insertion pathways (Figure 6). The first pathway follows the classic scheme that starts with soluble lysenin monomers binding sphingomyelin clusters. ${ }^{28}$ Those sphingomyelin clusters, such as lipid raft ${ }^{29,30}$, are used as platforms to enhance the monomer-monomer encounters, thus increasing the oligomerization rate. After the oligomerization, which gives rise to the so-called prepore state, the oligomer can be inserted spontaneously if there are no steric constraints. It is in such a crowded environment in which the prepore-to-pore transition is blocked. As we have demonstrated in this article, steric blockage can be overcome by the presence of physiological calcium concentrations. After pore formation, the pores in 
sphingomyelin cluster borders could overcame steric blockage, liberating space and starting, therefore, a cascade of insertions that in term liberate space in the cluster and allow new lysenin binding, thus increasing the overall density of lysenin. The alternative pathway shares the first steps but the prepore-to-pore transition takes places in the late endosome after endocytosis. Such endocytosis could be triggered by the increase of calcium in the cytoplasm induced by $\approx 13 \%$ of pores that seems to be inherent to lysenin oligomer assemblies (Figure 1).
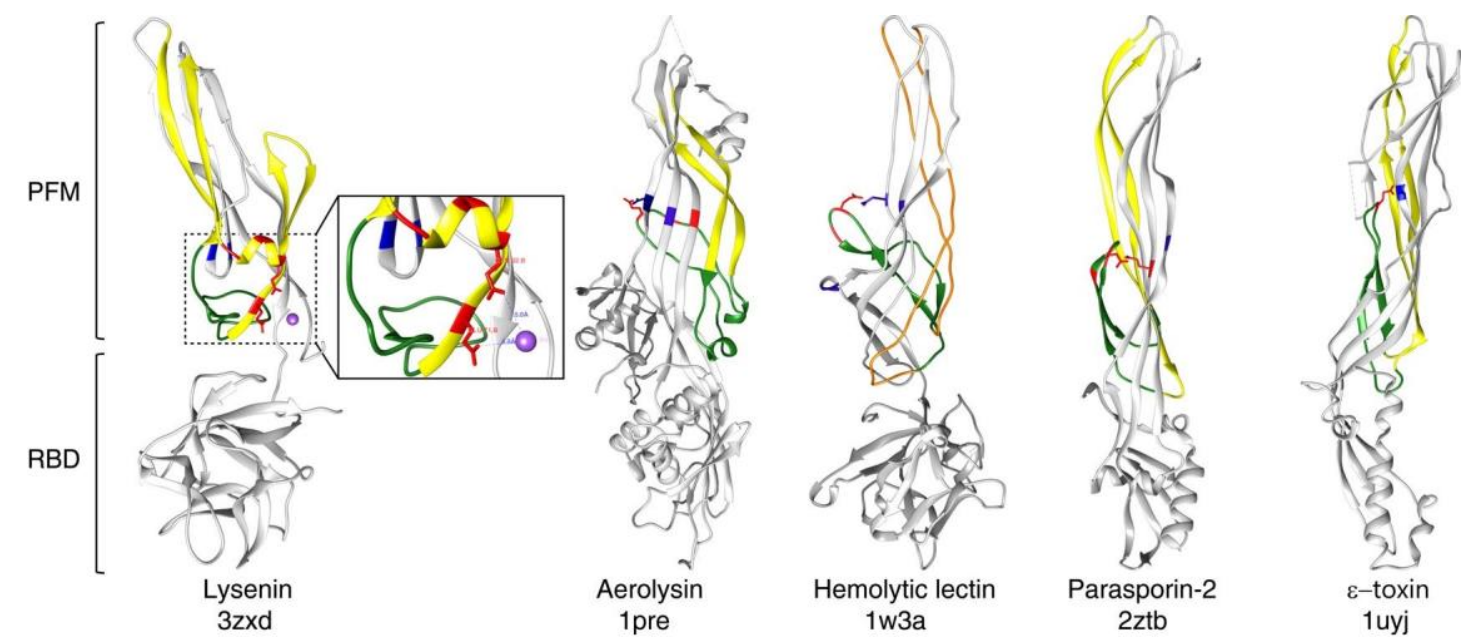

Figure 5 | Comparative structural approach of aerolysin family. Structural comparison of the $\mathrm{pH}$ sensor domain of lysenin soluble monomeric structure with four other members of aerolysin family. The $\beta$-hairpin (green) and the rest of the Pore Forming Module (PFM)(yellow) are colored to highlight them from the main body and the Receptor Binding Domain(RBD)(grey) following the nomenclature and colour scheme of Bokori-Brown et al. ${ }^{24}$. The $\mathrm{pH}$ receptor domain of lysenin is located in an $\alpha$-helix in which we identify three glutamic acids (Glu92, Glu94 and Glu97 highlighted in red) responsible for the $\mathrm{pH}$ response. In our model the acidic conditions charge residues Glu71 and Glu92 and by electrostatic repulsion with other charge residues (especially with Glu71, highlighted also in red, but also Asp121 and Asp126, highlighted in blue), resulting in an unfold of the region like a hinge. The Bhairpin of the rest of monomeric structures contains the Glutamic (red) or Aspartic (blue) acids identified as $\mathrm{pH}$ sensor domains in this study. We proposed for them a similar hinge working mechanism involving especially Glu252-Asp193 for aerolysin, Glu225-Asp305 for Hemolytic lectin, Glu183-Asp126 for Parasporin-2 and Glu137-Asp250 for $\varepsilon$-toxin. The supposed PFM of Hemolytic lectin was coloured in orange.

\section{Conclusions}

We found a new triggering mechanism of the prepore-to pore transition in lysenin induced by calcium cations. Such mechanism could be present in most PFTs as an alternative to the acidic insert activation. Our work can open new pathways in the understanding of the PFTs life cycle and, therefore, in the bacterial infection process. Deep understanding of this process is key to solve the arising problem of bacterial drug resistance. 


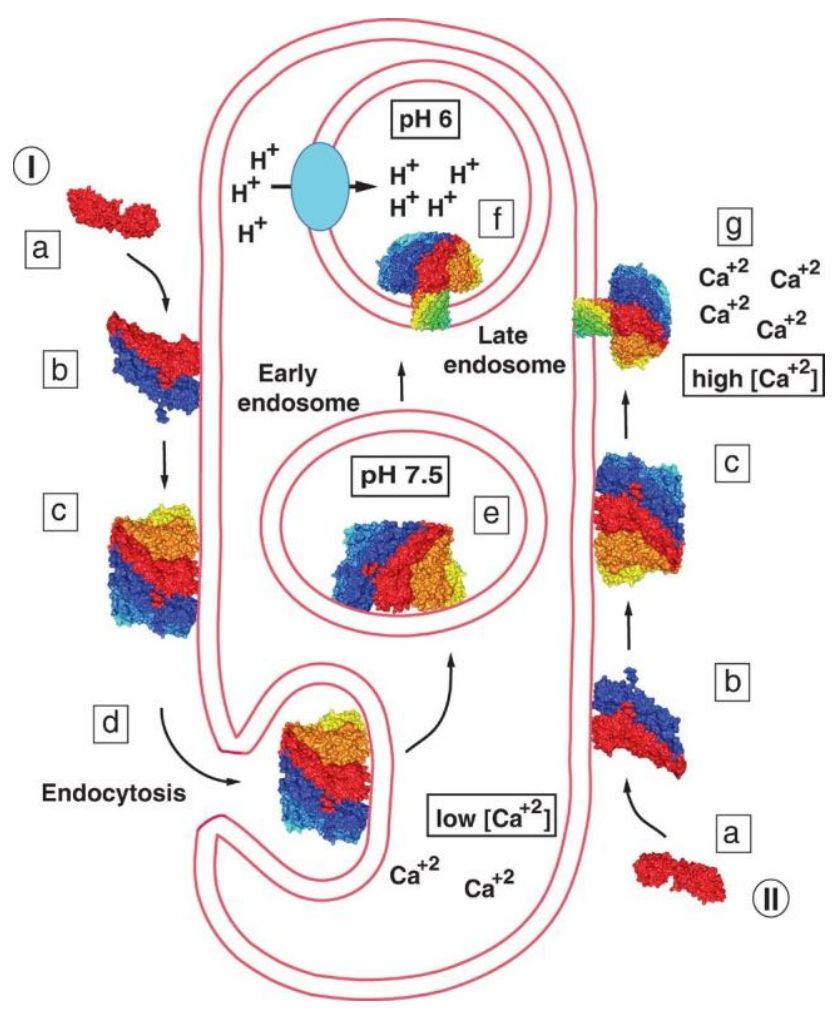

Figure 6 | Complete lysenin cycle. Two different pathways that end with lysenin pore are shown. The life cycle start with a soluble monomer (a) that bind specifically to sphingomyelin in the plasma membrane. After the binding the monomers interact with each other, (b) resulting in an oligomeric state called prepore (c). Those steps are common to both pathways. After the complete oligomerization, the first pathway (I) continues with an endocytosis process (d) that traps the prepore in an endosome (e). The proton pump reduces the $\mathrm{pH}$ values triggering lysenin insertion (f). In the second pathway, (II) lysenin insertion is triggered by high calcium concentration (g).

\section{Acknowledgements}

The authors would like to express their gratitude to Doctor Hirohide Takahashi. Others were involved in several degrees, our gratitude to all of them.

\section{Competing interest}

Author declare no competing interest. 


\section{Material and Methods}

\section{Protein origin}

Natural Lysenin from earthworm, Eisenia foetida, was obtained from Peptide Institute (Osaka, Japan). Lysenin mutant (E92, 94, 97Q) CDNA fragment was ordered from GenScript, USA. The cDNA fragments were subcloned into a $\mathrm{pET} 28 \mathrm{a}$ vector at BamHI and Hind III sites. This vector was transformed into BL21 (DE3) strain (New England BioLabs France, Evry, France). The transformed cells were inoculated into 1 liter of LB medium containing $50 \mu \mathrm{g} / \mathrm{ml}$ kanamycin sulfate, and incubated at $37^{\circ} \mathrm{C}$ while shaking at $220 \mathrm{rpm}$ until the $\mathrm{OD}_{600}$ value reached 0.6 . For induction of lysenin expression, isopropyl $\beta$-D1thiogalactopyranoside (IPTG) was added to the bacterial medium $(0.5 \mathrm{mM}$, final concentration) while shaking at $20^{\circ} \mathrm{C}$ and $200 \mathrm{rpm}$. The bacteria were collected by centrifugation at $2000 \mathrm{~g}$ for 10 minutes. The activity of proteases was inhibited by the addition of $1 \mathrm{mM}$ phenylmethane sulfonyl fluoride. To disrupt the bacteria we perform 3 sonication cycles with intervals of 30 seconds on ice. The resulted suspension was shaken at $4^{\circ} \mathrm{C}$ for 30 minutes in presence of $0.1 \%$ Triton X-100 and RNase/DNase at $10 \mathrm{\mu g} / \mathrm{ml}$ concentration. The crude extract was then centrifuged at $10,000 \times \mathrm{g}$ for 30 minutes. The supernatant (volume; typically up to $10 \mathrm{ml}$ ) was collected and mixed with $1 \mathrm{ml}$ of chelating Nickel Ni-NTA Affinity Resin (Generon, United Kingdom) in Phosphatebuffered saline (PBS) at pH7.5. Lysenin binding to the Ni-NTA resin was performed through 1 hour incubation at $4^{\circ} \mathrm{C}$ with gentle shaking, and the resin was washed with PBS containing $100 \mathrm{mM}$ imidazole- $\mathrm{HCl}, \mathrm{pH} 7.5$ for 3 times. The lysenin was eluted with $2 \mathrm{ml}$ of $250 \mathrm{mM}$ imidazole- $\mathrm{HCl}$. To eliminate the imidazole, the eluate was dialyzed against 1 liter of PBS at pH7.5 for overnight.

\section{Sample preparation for High-speed atomic force microscopy observation}

Egg Sphingomyelin (SM) and Cholesterol (chol) (Avanti Polar Lipids, Alabama, USA) at a molar ratio SM/Chol (1:1) were used to form giant unilamellar vesicles (GUVs) through electroswelling ${ }^{32}$. Of each lipid $10 \mu \mathrm{l}$ at $3 \mathrm{mM}$ dissolved in chloroform/methanol (3:1) were deposited in a glass plate coated with indium tin oxide with about $100 \Omega$ resistivity (Sigma-Aldrich) and placed 60 minutes in the desiccator for complete solvent evaporation. A U-shaped rubber piece of $\sim 1 \mathrm{~mm}$ thickness was sandwiched between two indium tin oxide coated slides. The so-formed chamber was filled with $\sim 400 \mu l$ of $200 \mathrm{mM}$ sucrose solution and exposed to $1.5 \mathrm{~V}$ sinusoidal $10 \mathrm{~Hz}$ AC current for 3 hours followed by squared $5 \mathrm{~Hz} \mathrm{AC}$ current for 15 minutes, at $55^{\circ} \mathrm{C}$. GUVs were harvested from the chamber. To form the supported lipid bilayers (SLBs) for HS-AFM, $1 \mu$ l of GUV solution was placed on a $1.5 \mathrm{~mm}$-diameter freshly cleaved mica disk covered with $1 \mu \mathrm{l}$ of Phosphate-buffered saline (PBS) and incubated for 30 minutes. To remove lipid that was not firmly attached the SLB was intensely rinsed with PBS. Once the bilayer was formed, $1 \mu \mathrm{l}$ of purified Lysenin was incubated for 15 minutes. Excess protein was again rinsed with PBS or physiological buffer. 


\section{High-speed atomic force microscopy}

HS-AFM movies were acquired with an Ando-type set-up ${ }^{33}$ equipped with a super luminescent diode (emission wavelength: 750 nm; EXS 7505-B001, Exalos, Schlieren, Switzerland) and a digital high-speed lock-in Amplifier (Hinstra, Transcommers, Budapest, Hungary). ${ }^{34} 8 \mu \mathrm{m}$-long cantilevers with spring constant $k=0.15 \mathrm{Nm}^{-1}$, resonance frequency $f_{(r)}=500-700 \mathrm{kHz}$ and quality factor $Q \approx 1$ in liquid (USC-1.2, NanoWorld, Neuchâtel, Switzerland), featuring an electron beam deposition (EBD) tip, were used. For high-resolution imaging the tip was sharpened by helium plasma etching using a plasma cleaner (Diener electronic, Ebhausen, Germany), resulting in a final tip radius tip of $\sim 2 \mathrm{~nm}$, as judged from analysis of the indentation inside the Lysenin rings. Amplitude modulation was used for imaging with free amplitude of $\sim 1.2 \mathrm{~nm}$ and operating set point amplitude of $\sim 0.9 \mathrm{~nm}$. Under this conditions we calculate the apply force following $F=\left(k k_{c c} / Q Q_{c c}\right)^{*}\left((1-\alpha)^{*} A A_{0}\left(A A_{0}-A_{s}{ }^{2} / A_{0}\right)^{1 / 2}\right.$ where $A_{0}$ is the free amplitude, $A s$ is the setpoint amplitude, and $\alpha \approx 0.5$ for short cantilevers, $\alpha$ being the ratio $(0<\alpha<1)$ of amplitude reduction caused by the cantilever resonance frequency shift over the total amplitude reduction. Under our imaging conditions $F=84 \mathrm{pN} .{ }^{35}$ All experiments were performed at room temperature and in PBS or physiological buffer (20mM HEPES, pH7.5, 120mM NaCl)

\section{High-speed atomic force microscopy image treatment}

Image treatment was limited to the correction of a first-order XY plane fit and XY drift correction of the HS-AFM movie. ${ }^{36}$

\section{Structure analysis}

Molecular graphics and analyses were performed with the UCSF Chimera package. ${ }^{37}$ 


\section{References}

1. Dal Peraro, M. \& van der Goot, F.G. Pore-forming toxins: ancient, but never really out of fashion. Nat Rev Microbiol 14, 77 (2016).

2. Iacovache, I., Bischofberger, M. \& van der Goot, F.G. Structure and assembly of pore-forming proteins. Curr Opin Struct Biol 20, 241 (2010).

3. Reboul, C.F., Whisstock, J.C. \& Dunstone, M.A. Giant MACPF/CDC pore forming toxins: A class of their own. Biochim Biophys Acta 1858, 475 (2016).

4. Ros, U. \& Garcia-Saez, A.J. More Than a Pore: The Interplay of Pore-Forming Proteins and Lipid Membranes. J Membr Biol 248, 545 (2015).

5. Young, J.A. \& Collier, R.J. Anthrax toxin: receptor binding, internalization, pore formation, and translocation. Annu Rev Biochem 76, 243 (2007).

6. Schnupf, P. \& Portnoy, D.A. Listeriolysin 0: a phagosome-specific lysin. Microbes Infect 9, 1176 (2007).

7. Nelson, L.D., Johnson, A.E. \& London, E. How interaction of perfringolysin 0 with membranes is controlled by sterol structure, lipid structure, and physiological low $\mathrm{pH}$ : insights into the origin of perfringolysin 0-lipid raft interaction. J Biol Chem 283, 4632 (2008).

8. Czajkowsky, D.M., Iwamoto, H., Cover, T.L. \& Shao, Z. The vacuolating toxin from Helicobacter pylori forms hexameric pores in lipid bilayers at low $\mathrm{pH}$. Proc Natl Acad Sci U S A 96, 5 (1999).

9. Sandvig, K. \& Olsnes, S. Diphtheria Toxin Entry into Cells is Facilitated by Low pH. The Journal of Cell Biology 87, 4 (1980).

10. Meusch, D., Gatsogiannis, C., Efremov, R.G., Lang, A.E., Hofnagel, O., Vetter, I.R., Aktories, K. \& Raunser, S. Mechanism of Tc toxin action revealed in molecular detail. Nature 508, 61 (2014).

11. Welch, R. Pore-forming cytolysins of gram-negative bacteria. Mol Microbiol 5, 521 (1991).

12. Tokunaga, H. \& Nakae, T. Calcium ion-mediated regulation of the $\alpha$-toxin pore of Staphylococcus aureus. Biochimica et Biophysica Acta (BBA)Biomembranes 1105, 125 (1992).

13. Unwin, P. \& Ennis, P. Two configurations of a channel-forming membrane protein. Nature 307, 609 (1984).

14. Sugawara, T., Yamashita, D., Kato, K., Peng, Z., Ueda, J., Kaneko, J., Kamio, Y., Tanaka, Y. \& Yao, M. Structural basis for pore-forming mechanism of staphylococcal $\alpha$-hemolysin. Toxicon 108, 226 (2015).

15. Koster, S., van Pee, K., Hudel, M., Leustik, M., Rhinow, D., Kuhlbrandt, W., Chakraborty, T. \& Yildiz, O. Crystal structure of listeriolysin 0 reveals molecular details of oligomerization and pore formation. Nat Commun $\mathbf{5}$, 3690 (2014).

16. Gaboriaud, C., Gregory-Pauron, L., Teillet, F., Thielens, N.M., Bally, I. \& Arlaud, G.J. Structure and properties of the $\mathrm{Ca}(2+)$-binding CUB domain, a 
widespread ligand-recognition unit involved in major biological functions. Biochem J 439, 185 (2011).

17. Sekizawa, Y., Hagiwara, K., Nakajima, T. \& Kobayashi, H. A Novel Protein, Lysenin, That Causes Contraction of the Isolated Rat Aorta : Its Puriification from the Coleomic Fluid of the Earthworm, Eisenia Foetida. Biomedical research 17, 197 (1996).

18. De Colibus, L., Sonnen, Andreas F.P., Morris, Keith J., Siebert, C.A., Abrusci, P., Plitzko, J., Hodnik, V., Leippe, M., Volpi, E., Anderluh, G. \& Gilbert, Robert J.C. Structures of Lysenin Reveal a Shared Evolutionary Origin for PoreForming Proteins And Its Mode of Sphingomyelin Recognition. Structure 20, 1498 (2012).

19. Ishitsuka, R. \& Kobayashi, T. Cholesterol and lipid/protein ratio control the oligomerization of a sphingomyelin-specific toxin, lysenin. Biochemistry 46, 1495 (2007).

20. Munguira, I., Casuso, I., Takahashi, H., Rico, F., Miyagi, A., Chami, M. \& Scheuring, S. Glasslike Membrane Protein Diffusion in a Crowded Membrane. ACS Nano 10, 2584 (2016).

21. Yilmaz, N., Yamada, T., Greimel, P., Uchihashi, T., Ando, T. \& Kobayashi, T. Real-Time Visualization of Assembling of a Sphingomyelin-Specific Toxin on Planar Lipid Membranes. Biophys J 105, 1397 (2013).

22. Podobnik, M., Savory, P., Rojko, N., Kisovec, M., Wood, N., Hambley, R., Pugh, J., Wallace, E.J., McNeill, L., Bruce, M., Liko, I., Allison, T.M., Mehmood, S., Yilmaz, N., Kobayashi, T., Gilbert, R.J., Robinson, C.V., Jayasinghe, L. \& Anderluh, G. Crystal structure of an invertebrate cytolysin pore reveals unique properties and mechanism of assembly. Nat Commun 7, 11598 (2016).

23. Munguira, I.L.B., Takahashi, H. \& I.Casuso. Crowding and pH roles in Lysenin toxin insertion. Nature Comunications, Under submision. (2017).

24. Bokori-Brown, M., Martin, T.G., Naylor, C.E., Basak, A.K., Titball, R.W. \& Savva, C.G. Cryo-EM structure of lysenin pore elucidates membrane insertion by an aerolysin family protein. Nat Commun 7, 11293 (2016).

25. Czajkowsky, D.M., Hotze, E.M., Shao, Z. \& Tweten, R.K. Vertical collapse of a cytolysin prepore moves its transmembrane b-hairpins to the membrane. The EMBO Journal 23, 3206 (2004).

26. Leung, C., Hodel, A.W., Brennan, A.J., Lukoyanova, N., Tran, S., House, C.M., Kondos, S.C., Whisstock, J.C., Dunstone, M.A. \& Trapani, J.A. Real-time visualization of perforin nanopore assembly. Nature Nanotechnology (2017).

27. Carafoli, E. \& Krebs, J. Why Calcium? How Calcium Became the Best Communicator. J Biol Chem 291, 20849 (2016).

28. Yamaji-Hasegawa, A., Hullin-Matsuda, F., Greimel, P. \& Kobayashi, T. Poreforming toxins: Properties, diversity, and uses as tools to image sphingomyelin and ceramide phosphoethanolamine. Biochimica et Biophysica Acta (BBA)-Biomembranes 1858, 576 (2016). 
29. Lafont, F., Abrami, L. \& van der Goot, F.G. Bacterial subversion of lipid rafts. Curr Opin Microbiol 7, 4 (2004).

30. Iacovache, I., van der Goot, F.G. \& Pernot, L. Pore formation: An ancient yet complex form of attack. Biochimica et Biophysica Acta (BBA) Biomembranes 1778, 1611 (2008).

31. Simons, K. \& Gerl, M.J. Revitalizing membrane rafts: new tools and insights. Nature Reviews Molecular Cell Biology 11, 688 (2010).

32. Angelova, M.I. \& Dimitrov, D.S. Liposome electroformation. Faraday discussions of the Chemical Society 81, 303 (1986).

33. Ando, T., Kodera, N., Takai, E., Maruyama, D., Saito, K. \& Toda, A. A highspeed atomic force microscope for studying biological macromolecules. Proceedings of the National Academy of Sciences 98, 12468 (2001).

34. Colom, A., Casuso, I., Rico, F. \& Scheuring, S. A hybrid high-speed atomic force-optical microscope for visualizing single membrane proteins on eukaryotic cells. Nat Commun 4(2013).

35. Ando, T., Uchihashi, T. \& Scheuring, S. Filming biomolecular processes by high-speed atomic force microscopy. Chem Rev 114, 3120 (2014).

36. Husain, M., Boudier, T., Paul-Gilloteaux, P., Casuso, I. \& Scheuring, S. Software for drift compensation, particle tracking and particle analysis of high-speed atomic force microscopy image series: SOFTWARE FOR HIGHSPEED ATOMIC FORCE MICROSCOPY IMAGE SERIES. Journal of Molecular Recognition 25, 292 (2012).

37. Pettersen, E.F., Goddard, T.D., Huang, C.C., Couch, G.S., Greenblatt, D.M., Meng, E.C. \& Ferrin, T.E. UCSF Chimera-a visualization system for exploratory research and analysis. J Comput Chem 25, 1605 (2004). 